\title{
Prevalencia de Helicobacter pylori y características histopatológicas en biopsias gástricas de pacientes con síntomas dispépticos en un centro de referencia de Medellín
}

\author{
Prevalence of Helicobacter pylori and Histopathological Features in Gastric \\ Biopsies from Patients with Dyspeptic Symptoms at a Referral Center in Medellin
} \author{
Santiago Estrada M., MD. ${ }^{5}$ \\ Estudiante de medicina, Universidad de Antioquia. \\ Medellín, Colombia. \\ 2 Estudiante de medicina, Universidad Pontificia \\ Bolivariana. Medellín, Colombia. \\ 3 Médico patólogo, laboratorio clínico VID, Obra de la \\ Congregación Mariana. Profesor titular Universidad \\ de Antioquia. Medellín, Colombia. \\ ${ }^{4}$ Médico salubrista epidemiólogo. Grupo académico \\ de epidemiología clínica - GRAEPIC. Profesor \\ Universidad de Antioquia. Medellín, Colombia. \\ 5 Médico especialista en microbiología y parasitología \\ médicas. Director laboratorio clínico VID, Obra de la \\ Congregación Mariana. Medellín, Colombia. \\ Este trabajo tiene el respaldo académico y científico \\ de la Asociación Colombiana de Infectología y \\ fue presentado en el IX Encuentro Nacional de \\ Investigación en Enfermedades Infecciosas, el cual \\ se llevó a cabo del 15 al 17 de agosto del 2014 en la \\ ciudad de Barranquilla.
}

Simón Correa G., ${ }^{1}$ Andrés Felipe Cardona A., ${ }^{1}$ Tomás Correa G., ${ }^{2}$ Luis Alfonso Correa L., MD, ${ }^{3}$ Héctor Iván García G., MD, MSc, ${ }^{4}$

Fecha recibido: $19-05-15$ Fecha aceptado: 26-01-16

\section{Palabras clave}

\begin{abstract}
Resumen
Introducción: Helicobacter pylori produce la infección crónica más prevalente en el mundo, principalmente en países en desarrollo. Objetivo: el principal objetivo de este estudio fue estimar la prevalencia de la infección por H. pylori y gastritis en pacientes con síntomas dispépticos llevados a endoscopia digestiva superior y correlacionarlos con los principales hallazgos histopatológicos. Métodos: se revisaron 2708 biopsias gástricas de pacientes que consultaron por síntomas dispépticos entre el año 2012 y 2013 en la Clínica Diagnóstica Especializada VID de la Congregación Mariana de Medellín, y cuyas biopsias se estudiaron en el laboratorio de dicha institución. Las variables histológicas de los resultados reportados por el patólogo se analizaron con métodos estadísticos. Resultados: la prevalencia de la infección por $H$. pylori fue del $36,4 \%$; la media de edad de los pacientes infectados fue de 46,5 años (DE 17,1), con un pico de prevalencia en el grupo de 40-49 años, a partir del cual disminuyó. La cantidad de $H$. pylori se correlacionó con la intensidad de la inflamación y de la actividad; asimismo, la presencia de la bacteria se asoció con metaplasia, folículos linfoides, atrofia y pólipos hiperplásicos. La intensidad de la inflamación se asoció con la cantidad de $H$. pylori y la actividad neutrofílica. Conclusión: la prevalencia de la infección por $H$. pylori en este estudio es baja comparada con otras investigaciones, y exhibió un comportamiento inusual en los grupos de edad descritos. La cantidad de H. pylori se correlacionó con la intensidad de la inflamación y de la actividad neutrofílica.
\end{abstract}

Helicobacter pylori, prevalencia, gastritis, atrofia gástrica, metaplasia intestinal, displasia, cáncer gástrico.

\begin{abstract}
Introduction: Helicobacter pylori infection is considered to be the most prevalent chronic infection in the world. It occurs primarily in developing countries. Objective: The main objective of this study was to estimate the prevalence of $H$. pylori infections and gastritis in patients with dyspeptic symptoms who underwent upper endoscopy and correlate endoscopic findings with principal histopathological findings. Methods: We reviewed 2,708 gastric biopsies from patients who had come to the VID Specialized Diagnostic Clinic of the Congregación Mariana in Medellín because of dyspeptic symptoms in 2012 and 2013. Biopsies were studied in the VID Clinical Laboratory of the Congregación Mariana. Histological variables of the results reported by the pathologist were analyzed with statistical methods. Results: The prevalence of $H$. pylori infection was $36.4 \%$, the average age of infected patients was 46.5 years (SD: 17.1) with a peak prevalence in the group between 40 and 49 years. Prevalence decreased after age 49 . The amount of $H$. pylori was correlated with the intensity of inflammation and activity. The presence of the bacteria was associated with the presence of metaplasia, lymphoid follicles, atrophy and hyperplastic polyps. The intensity of inflammation was associated with the amount of $H$. pylori and neutrophil activity. Conclusion: The prevalence of $H$. pylori infection in our study is low compared with other research and exhibited unusual behavior by age group. The amount of $H$. pylori was correlated with the intensity of inflammation and neutrophil activity.
\end{abstract}

Keywords

Helicobacter pylori, prevalence, gastritis, gastric atrophy, intestinal metaplasia, dysplasia, gastric cáncer. 


\section{INTRODUCCIÓN}

H. pylori es un bacilo gramnegativo, microaerófilo, con 4 a 6 flagelos, identificado por primera vez en 1983 por Warren y Marshall, y asociado con la enfermedad ácido péptica. Su prevalencia es alta, especialmente en países en vía de desarrollo, y aumenta con la edad; además, es considerada la infección bacteriana crónica más común en el mundo (1). $H$. pylori ingresa al tracto digestivo, presumiblemente por vía fecal-oral u oral-oral, y genera proteasas que debilitan el revestimiento mucoso del epitelio gástrico, así como expresa ureasa para desdoblar la urea de la luz gástrica en amonio y dióxido de carbono y, de esta manera, genera una capa que le provee protección frente al $\mathrm{pH}$ ácido del estómago. Por medio de proteínas específicas, se adhiere a la membrana de las células epiteliales gástricas, principalmente a aquellas del antro, ya que allí no hay secreción ácida, si bien este puede encontrarse distribuido en todo el estómago (2-5). Al adherirse al epitelio puede generar una reacción inflamatoria con migración de polimorfonucleares y linfocitos, que a su vez conllevarán a la generación de folículos linfoides característicos de esta infección.

Factores propios de la bacteria, del hospedero, y la cronicidad de la infección contribuyen a la aparición de úlceras gástricas o duodenales que pueden complicarse con hemorragia o perforaciones (5-6). Otras enfermedades asociadas con la infección por H. pylori son el cáncer gástrico y el linfoma asociado con el tejido linfoide de la mucosa (MALT), por lo cual la Organización Mundial de la Salud (OMS) la ha clasificado como carcinogénica (7-9). La magnitud de la infección aún no se ha relacionado con el desarrollo de úlceras o de las otras enfermedades citadas (7).

La infección es asintomática en la mayoría de los casos, no obstante, pueden presentarse síntomas clásicos de la enfermedad ácido péptica, entre ellos epigastralgia, saciedad temprana, sensación de vacío o hambre dolorosa, náuseas, vómito y en caso de sangrado, melenas, hematemesis y anemia $(7,10)$. El diagnóstico puede confirmarse con diferentes pruebas que incluyen urea en aliento, serología, detección de antígeno en heces, ureasa rápida en biopsia, estudio histopatológico y/o cultivo; todas ellas con muy buena sensibilidad y especificidad. El objetivo del tratamiento es la erradicación de la bacteria (11). Debido a la facilidad del diagnóstico, a la efectividad de la terapia y a los desenlaces fatales que se pueden evitar con la erradicación de esta bacteria, es muy importante conocer la prevalencia de esta infección en nuestro medio.

Los objetivos de este estudio son: (i) estimar la prevalencia de la infección por $H$. pylori, (ii) describir las características histopatológicas, y (iii) correlacionar la infección por H. pylori con hallazgos histopatológicos de las biopsias gástricas de pacientes con síntomas dispépticos tomadas por endoscopia y analizadas en un laboratorio de referencia de Medellín.

\section{MATERIALES Y MÉTODOS}

Se realizó un estudio descriptivo retrospectivo. La población fueron los pacientes atendidos en la Clínica Diagnóstica VID, quienes consultaron por síntomas dispépticos y a quienes se les practicó endoscopia digestiva superior y biopsia en el periodo comprendido entre mayo de 2012 y abril de 2013. Se excluyeron aquellos pacientes que habían recibido terapia antimicrobiana para $H$. pylori y aquellos que habían recibido terapia supresora de ácido.

En la sección de Patología del laboratorio clínico VID se recopilaron las biopsias gástricas de estos pacientes. Las placas fueron interpretadas nuevamente por el patólogo del laboratorio, quien diligenció un formulario de variables histológicas para cada caso. La información recolectada se transcribió en una base de datos por duplicado; luego, ambas bases fueron comparadas en el software Microsoft Excel Compare y los registros inconsistentes entre ambas se corrigieron con los formatos de variables histológicas originales.

Las variables histológicas recopiladas fueron: H. pylori, inflamación, actividad (neutrófilos), erosión, úlcera, eosinófilos, metaplasia, folículos linfoides, carcinoma, pólipos hiperplásicos y linfoma. Las 3 primeras variables se categorizaron de acuerdo con el sistema actualizado de Sydney en negativo, leve, moderado y marcado (12); las variables histológicas restantes se calificaron como presente o ausente.

Las muestras de patología se fijaron en formol deshidratado y bufferado al $10 \%$ y se incluyeron en bloques de parafina a las 12 horas; los tejidos fueron orientados ubicando la mucosa en un plano perpendicular a la superficie del corte. De cada fragmento se obtuvieron cortes histológicos de 4 micrómetros de espesor, los cuales se montaron en láminas de vidrio en múltiples grupos y se colorearon con hematoxilina eosina para su lectura.

Para la descripción estadística se usaron distribuciones de frecuencia, promedios y desviaciones estándar; para las correlaciones entre las variables ordinales se usó el coeficiente de Spearman, y para las asociaciones entre variables categóricas se usó la prueba $\mathrm{Chi}^{2}$ o la prueba exacta de Fischer. Para la significación estadística se usó un valor $\mathrm{p}$ $=0,01$. Todos los análisis se realizaron con el software SPSS 18 (IBM, Armonk, New York).

\section{RESULTADOS}

\section{Características demográficas}

En total se analizaron 2708 muestras correspondientes a igual número de pacientes, de los cuales 1727 (63,8\%) 
fueron mujeres y $981(36,2 \%)$ hombres. La edad promedio de la población total fue 51,3 años (DE 17,9); para el sexo masculino la media fue de 50,2 años (DE 17,6) y para el femenino fue de 52 (DE 18,1). La distribución por grupos de edad de las biopsias gástricas analizadas se observa en la tabla 1 .

\section{Prevalencia de $\boldsymbol{H}$. pylori}

Se encontró $H$. pylori en 986 muestras de pacientes $(36,4 \%)$. En el $20,3 \%$ la infección se clasificó como leve y en el 16,1\% como marcada; no se encontraron casos moderados. La prevalencia de $H$. pylori por sexo fue del $34,6 \%$ en las mujeres y del 39,6\% en los hombres. El promedio de edad de los pacientes con $H$. pylori fue de 46,5 años (DE 17,1); la distribución de H. pylori por grupo de edad y sexo se observa en la tabla 1.

Tabla 1. Distribución de Helicobacter pylori por grupos de edad y sexo.

\begin{tabular}{lccc}
\hline Grupo de edad & \multicolumn{2}{c}{ Sexo } & Total \\
\cline { 2 - 3 } & Femenino & Masculino & \\
\hline Menor de 20 & 26 & 18 & 44 \\
& $4,4 \%$ & $4,6 \%$ & $4,5 \%$ \\
$20-29$ & 99 & 56 & 155 \\
& $16,6 \%$ & $14,4 \%$ & $15,7 \%$ \\
$30-39$ & 85 & 69 & 154 \\
& $14,2 \%$ & $17,8 \%$ & $15,6 \%$ \\
$40-49$ & 113 & 91 & 204 \\
& $18,9 \%$ & $23,5 \%$ & $20,7 \%$ \\
$50-59$ & 111 & 70 & 181 \\
& $18,6 \%$ & $18,0 \%$ & $18,4 \%$ \\
$60-69$ & 103 & 47 & 150 \\
& $17,2 \%$ & $12,1 \%$ & $15,2 \%$ \\
70 o mayor & 61 & 37 & 98 \\
& $10,2 \%$ & $9,5 \%$ & $9,9 \%$ \\
\hline Total & 598 & 388 & 986 \\
\hline
\end{tabular}

\section{Hallazgos histopatológicos}

Inflamación (gastritis): se encontró inflamación en el 99,9\% de los casos. La inflamación leve predominó en 2211 de los casos $(81,6 \%), 491(18,1 \%)$ presentaron inflamación moderada y $2(0,1 \%)$ presentaron inflamación marcada; no se presentó infiltrado inflamatorio en 4 pacientes. La media de edad para la gastritis fue de 51,3 años (DE 17,9).

Actividad neutrofílica: en total se registraron 1014 casos $(37,4 \%)$ con actividad neutrofílica; de estos, 523 (19,3\%) correspondieron a casos leves, $490(18,1 \%)$ a moderados y solo 1 caso con intensidad marcada.
Otros hallazgos histológicos: la prevalencia de otros hallazgos fue: erosión $2,1 \%$, úlcera $1,3 \%$, metaplasia $13,3 \%$, atrofia $1,7 \%$, carcinoma $0,6 \%$, folículos linfoides $8,1 \%$ y pólipos hiperplásicos 3,3\%. Solo se documentó 1 caso de linfoma.

\section{Correlación y asociación de $\boldsymbol{H}$. pylori con otras variables}

La cantidad de H. pylori y la intensidad de la inflamación (categorizadas en negativa, leve, moderada o marcada) tuvieron correlación (coeficiente de Spearman 0,7, p $<0,01)$; la intensidad de la actividad de los neutrófilos y la cantidad de H. pylori también se correlacionó (coeficiente de Spearman 0,96, p <0,01).

Se encontró asociación significativa entre la presencia de $H$. pylori y la presencia de metaplasia $(\mathrm{p}<0,01)$, folículos linfoides $(\mathrm{p}<0,01)$, atrofia $(\mathrm{p}<0,01)$ y pólipos hiperplásicos $(p<0,01)$. No hubo asociación significativa entre la presencia de $H$. pylori y erosión ( $\mathrm{p}>0,01)$, úlcera gástrica ( $p>0,01)$, eosinófilos $(p>0,01)$ y carcinoma $(p>0,01)$.

\section{Correlación y asociación de la inflamación con otras variables}

La intensidad de la inflamación tuvo una correlación lineal positiva con la intensidad de la actividad neutrofílica ( $\mathrm{p}$ $<0,01)$. No se encontró asociación entre la presencia de inflamación y otras variables.

\section{DISCUSIÓN}

La infección por H. pylori ha sido demostrada en todo el mundo y afecta a todos los grupos de edad; se estima que el $50 \%$ de la población está infectada (13). En este estudio se encontró $H$. pylori en el $36,4 \%$ de los casos.

Al comparar resultados, se puede demostrar que la prevalencia es baja comparada con otras investigaciones. El estudio de prevalencia de $H$. pylori en Colombia, publicado en 2003 por Bravo y colaboradores, la estimó en el 69,1\% de 8652 biopsias gástricas provenientes de 16 ciudades del país; la ciudad de Medellín, en esta serie, presentó una prevalencia del $65 \%$, casi el doble de la encontrada en nuestra serie. Otras ciudades con una mayor prevalencia fueron, en su orden, Tunja $(99,1 \%)$, Popayán $(86,5 \%)$ y Manizales $(85,5 \%)(14)$.

En un estudio publicado por Garg y colaboradores se reportó una prevalencia de la infección del $43 \%$ en un centro en India, sin embargo, otros estudios provenientes del mismo país han reportado prevalencias tan altas como del $78 \%$ y $65 \%$ (15-17). Se ha descrito que, en países en vías de desarrollo, más del 50\% de la población está infectada a los 
10 años de edad, con un pico de prevalencia del $80 \%$ justo antes de los 50 años $(13,18)$; en contraste, en los países desarrollados tan solo el $10 \%$ de la población está infectada a los 10 años de edad (19) y aumenta desde un $10 \%$ entre los 18 y 30 años de edad hasta un $50 \%$ en los mayores de 60 años (13). En nuestra serie se encontró que, en los menores de 60 años, la prevalencia de la infección fue de $41,9 \%$, y de $26,1 \%$ en los mayores de 60 años.

La prevalencia de la infección por $H$. pylori se relaciona con 3 factores: primero, la tasa de adquisición de la misma, es decir, la incidencia; segundo, la tasa de pérdida de la infección; y, tercero, la persistencia prolongada de la bacteria en la mucosa gastroduodenal entre infección y erradicación (13). La tabla 2 muestra la prevalencia de la infección en diferentes estudios.

Torres y colaboradores encontraron asociación entre infección y hacinamiento [OR $=1,4$ (IC 95\%: 1,23-1,60)], bajo nivel educativo $[\mathrm{OR}=2,42$ (IC 95\%: $1,71-3,44)$ ) y bajo nivel socioeconómico [OR =1,43 (IC 95\%: 1,26-1,63)] (18); esto podría explicar, en parte, la baja prevalencia de $H$. pylori encontrada en la presente serie, pues la población que consulta el laboratorio es predominantemente de clase media y media-alta. Otros factores asociados con tasas más altas de infección son el número de hijos, las camas compartidas y la calidad de las aguas (20-22); es de tener en cuenta que el acueducto de la ciudad de Medellín está clasificado con altos estándares de calidad de consumo (23). Además, se ha propuesto que la disminución en la prevalencia de la infección va paralela con el mejoramiento de la economía, de acuerdo con un estudio japonés que analizó la prevalencia de la infección en la pre y posguerra (24); esto se podría plantear como una posible explicación en el descenso de la prevalencia del año 2003 visto en el estudio de Bravo y colaboradores (de 65\%) a la actual prevalencia del presente estudio (36,4\% 10 años después).
Clásicamente, se ha descrito que la prevalencia de la infección aumenta con la edad (1). Es así como Pounder y colaboradores, en un estudio que evaluó la prevalencia de $H$. pylori en diferentes países, la clasificó en 2 grupos: grupo 1 , aquellos países donde la mayoría de individuos se infectaban durante la niñez y la infección continuaba durante la edad adulta; grupo 2 , solo una minoría de los individuos se infectaban durante la niñez, pero la prevalencia de la infección aumentaba en proporción con la edad durante la vida adulta (13). La prevalencia de la infección exhibió un comportamiento inusual en nuestra serie. En la población general y para ambos sexos la prevalencia fue mayor en el grupo de 40 a 49 años, y partir de allí disminuyó progresivamente en los siguientes grupos de edad, tanto para la población general como para hombres y mujeres; la prevalencia fue mayor en hombres que en mujeres (39,6\% versus $34,6 \%)$, lo que coincide con los resultados de Dorji y colaboradores $(18,25)$.

En el 99,9\% de los pacientes se encontró gastritis, con una edad media de 51,3 años; de estos, solo el $36,4 \%$ se encontró infectado por H. pylori, resultado que no coincide con lo informado en la literatura, donde esta infección es una de las principales causas de esta enfermedad (2, 10, 32-34). El estudio colombiano de Bravo y colaboradores encontró una prevalencia de gastritis del 83,6\%, pero en Medellín fue significativamente más baja: $73,7 \%$ (14). El grupo de edad con mayor número absoluto de casos de gastritis fue el de 50-59 años, resultados similares a los encontrados en la literatura (15).

$\mathrm{La}$ atrofia glandular, la metaplasia intestinal y la displasia son lesiones de gran importancia clínica, pues se consideran precursoras de malignidad de acuerdo con la secuencia descrita por el Dr. Pelayo Correa (35); la prevalencia de atrofia y displasia fueron muy bajas, $1,7 \%$ y $0,1 \%$, respectivamente. Sin embargo, la prevalencia de metaplasia fue considerablemente más alta, del 13,3\%, similar a lo descrito en la India

Tabla 2. Prevalencia de Helicobacter pylori en diferentes estudios.

\begin{tabular}{|c|c|c|c|c|c|}
\hline Autor & Año & $\begin{array}{l}\text { Número de } \\
\text { pacientes }\end{array}$ & Prueba de laboratorio utilizada & Prevalencia & País \\
\hline Lim SH y col (26) & 2013 & 19272 & Serología lgG para H. pylori & $54,4 \%$ & Corea \\
\hline Bravo LE y col (14) & 2003 & 8652 & Biopsia para histopatología & $69,1 \%$ & Colombia \\
\hline Hu D y col (27) & 2013 & 3995 & Serología lgG para H. pylori & $44,9 \%$ & China \\
\hline Nakajima S y col (28) & 2010 & 1246 & Biopsia para histopatología & $52,7 \%$ & Japón \\
\hline Gill HH y col (17) & 1993 & 526 & Biopsia para histopatología y ureasa en biopsia & $65 \%$ & India \\
\hline Shiota S y col (29) & 2013 & 381 & Serología IgG para $H$. pylori & $71,1 \%$ & Bután \\
\hline Garg B y col (15) & 2012 & 300 & Biopsia para histopatología & $43 \%$ & India \\
\hline Krashias G y col (30) & 2013 & 103 & PCR para H. pylori en muestra de tejido & $39,8 \%$ & Chipre \\
\hline Bakri MM (31) & 2012 & 70 & Cultivo y PCR para H. pylori en muestra de tejido & $85,7 \%$ & Arabia Saudí \\
\hline
\end{tabular}

PCR: reacción en cadena de la polimerasa. 
con $14 \%$ de prevalencia (15). En el estudio de Bravo y colaboradores se encontró una prevalencia de metaplasia del $10,3 \%$ para hombres y $11 \%$ para mujeres (14).

Se encontraron 34 úlceras gástricas correspondientes al 1,3\% de casos, de los cuales 11 tenían $H$. pylori (32,3\%), siendo similar a lo descrito en Colombia y el mundo $(1,14)$.

En 1994, la Agencia Internacional para la Investigación del Cáncer incluyó al H. pylori en el grupo I de carcinógenos humanos para adenocarcinoma gástrico (36); la prevalencia de cáncer gástrico en esta serie fue solo del 0,6\%, considerablemente baja si se compara con la del estudio nacional de 2003 que fue del 9,3\% (14).

Varios estudios han demostrado la asociación entre $H$. pylori y el maltoma; se considera que la estimulación persistente de células $\mathrm{B}$ activadas por células $\mathrm{T}$ predispone al desarrollo de la neoplasia, y que el desarrollo de folículos linfoides antecede a la misma (37-39). En nuestra serie se encontraron 219 casos con folículos linfoides que corresponden al 8,1\%, aproximadamente la mitad de lo reportado en la literatura (15), y se encontró 1 caso de maltoma.

Asimismo, la cantidad de $H$. pylori se correlacionó positivamente con la intensidad de la inflamación y de la actividad; esto coincide con los hallazgos de Garg y Mysorekar $(15,40)$. Además, la presencia del microorganismo se asoció con la presencia de metaplasia, folículos linfoides, atrofia y pólipos hiperplásicos; es importante mencionar que $H$. pylori no coloniza el epitelio metaplásico, y la asociación positiva entre estas variables no refleja la invasión del epitelio con metaplasia por la bacteria sino la posibilidad de encontrar en una misma muestra áreas de metaplasia sin invasión y áreas sin metaplasia colonizadas. Por otra parte, se observó que con mayor intensidad de la inflamación, mayor intensidad de la actividad neutrofílica y más cantidad de H. pylori, similar a lo descrito en la literatura (15).

Es de anotar que este es un estudio de prevalencia de H. pylori y de hallazgos histopatológicos en una población seleccionada y sintomática; además, la presencia de $H$. pylori no siempre se asocia con síntomas dispépticos (es posible el estado de colonización en ausencia de síntomas), sin embargo, todos los pacientes evaluados presentaban dichos síntomas. Se considera que no es posible atribuir la universalidad de la sintomatología dispéptica a $H$. pylori, pues solo se encontró la presencia de la bacteria en el $36,4 \%$ de los casos.

En conclusión, la prevalencia de infección por H. pylori en este estudio es baja comparada con otras investigaciones, $y$ exhibió un comportamiento inusual en los grupos de edad descritos; por lo contrario, la prevalencia de gastritis es casi universal, mientras que la de metaplasia y folículos linfoides es similar a lo descrito en la literatura. Algunas de estas alte- raciones se correlacionan entre sí y son precursoras de malignidad, principalmente de adenocarcinomas y maltomas, causas importantes de morbilidad y mortalidad. La detección temprana y el tratamiento de las lesiones precursoras podrían impactar significativamente estos desenlaces.

\section{Agradecimientos}

Laboratorio clínico VID, Obra de la Congregación Mariana, por facilitar la información de la base de datos de los pacientes.

\section{Fuentes de financiación}

Estrategia de Sostenibilidad 2013-2014, Universidad de Antioquia.

\section{Conflicto de intereses}

Los autores declaran no tener ningún conflicto de intereses.

\section{REFERENCIAS}

1. Mandel GL, Bennet JE, Dolin R. Mandell, Couglas, and Bennett's Principles and Practice of Infectious Diseases. 7 ed. Philadelphia: Churchill Livingstone; 2010.

2. Ramírez A, Mendoza D, Ley J, Guerra J. Estudio del Helicobacter pylori en el Perú. Rev Peru Med Exp Salud Pública. 2002;19(4):209-14.

3. Logan RP. Adherence of Helicobacter pylori. Aliment Pharmacol Ther. 1996; 10 Suppl 1:3.

4. Dytoc M, Gold B, Louie M, Huesca M, Fedorko L, Crowe $\mathrm{S}$, et. al. Comparison of Helicobacter pylori and attachingeffacing Escherichia coli adhesion to eukaryotic cells. Infect Immun. 1993;61(2):448.

5. Kusters JG, van Vliet AH, Kuipers EJ. Pathogenesis of Helicobacter pylori infection. Clin Microbiol Rev. 2006;19(3):449.

6. Malfertheiner P, Chan F, McColl K. Peptic ulcer disease. Lancet. 2009;374:1449-61.

7. Parsonnet J, Friedman GD, Vandersteen DP, Chang Y, Vogelman JH, Orentreich $\mathrm{N}$, et al. Helicobacter pylori infection and risk of gastric carcinoma. $N$ Eng J Med. 1991;325:1127-31.

8. Romo C. Coria R. Helicobacter pylori, un modelo de bacteria carcinogénica. Rev Especialidades Médico-Quirúrgicas 2010;15(4):242-251.

9. Eck M, Schmausser B, Haas R, Greiner A, Czub S, MüllerHermelink HK. MALT-type lymphoma of the stomach is associated with Helicobacter pylori strains expressing the CagA protein. Gastroenterology. 1997;112(5):1482. 
10. Kenneth EL. Helicobacter pylori Infection. N Engl J Med. 2010;362:1597-604.

11. Malfertheiner P, Megraud F, O’Morain CA, Atherton J, Axon AT, Bazzoli F, et al. Management of Helicobacter pylori infection-the Maastricht IV/ Florence Consensus Report. Gut. 2012 May;61(5):646-64. doi: 10.1136/ gutjnl-2012-302084.

12. Dixon MF, Genta RM, Correa P. Classification and grading of gastritis. The updated Sydney System. International Workshop on the Histopathology of Gastritis, Houston 1994. Am J Surg Pathol. 1996 Oct;20(10):1161-81.

13. Pounder RE. The prevalence of Helicobacter pylori infection in different countries. Aliment Pharmacol Ther. 1995;9 Suppl 2:33.

14. Bravo LE, Cortes A, Carrascal E, Jaramillo R, Garcia LE, Bravo $\mathrm{PE}$ et al. Helicobacter pylori: patología y prevalencia en biopsias gástricas en Colombia. Colomb Med. 2003;34:124-134.

15. Garg B, Sandhu V, Sood N, Sood A, Malhotra V. Histopathological analysis of chronic gastritis and correlation of pathological features with each other and with endoscopic findings. Pol J Pathol. 2012;3:172-178.

16. Kumar A, Bansal R, Pathak VP, Kishore S, Arya PK. Histopathological changesin gastric mucosa colonized by H. pylori. Indian J Pathol Microbiol. 2006;49(3):352-356.

17. Gill HH, Desai HG, Majmudar P, Mehta PR, Prabhu SR. Epidemiology of Helicobacter pylori: The Indian scenario. Indian J Gastroenterol. 1993;12:9-11.

18. Torres J, Leal-Herrera Y, Perez-Perez G, Gomez A, Camorlinga-Ponce M, Cedillo-Rivera R, et al. A community-based seroepidemiologic study of Helicobacter pylori infection in Mexico. SOJ Infect Dis. 1998;178(4):1089.

19. Taylor DN, Blaser MJ. The epidemiology of Helicobacter pylori infection. Epidemiol Rev. 1991;13:42-49.

20. Hunt RH, Sumanac K, Huang JQ. Review article: Should we kill or should we save Helicobacter pylori? Aliment Pharmacol Ther. 2001; 15Suppl 1:51.

21. Webb PM, Knight T, Greaves S, Wilson A, Newell DG, Elder J, et al. Relation between infection with Helicobacter pylori and living conditions in childhood: Evidence for person to person transmission in early life. SOBMJ. 1994;308(6931):750.

22. Kivi M, Johansson AL, Reilly M, Tindberg Y. Helicobacter pylori status in family members as risk factors for infection in children. Epidemiol Infect. 2005;133(4):645.

23. Empresas Públicas de Medellín (EPM) [homepage en internet]. Medellín; Empresas Públicas de Medellín [actualizada 10 de julio de 2014, consultada 15 de julio de 2014]. Disponible en: http://www.epm.com.co/site/clientes_usuarios/Clientesyusuarios/Hogaresypersonas/Agua/ Indicadoresdecalidad.aspx

24. Asaka M, Kimura T, Kudo M, Takeda H, Mitani S, Miyasaki $\mathrm{T}$, et al. Relationship of Helicobacter pylori to serum pepsinogens in an asymptomatic Japanese population. Gastroenterology. 1992;102(3):760.
25. Dorji D1, Dendup T, Malaty HM, Wangchuk K, Yangzom D, Richter JM. Epidemiology of Helicobacter pylori in Bhutan: the role of environment and Geographic location. Helicobacter. 2014;19(1):69-73.

26. Lim SH, Kwon JW, Kim N, Kim GH, Kang JM, Park MJ, et al. Prevalence and risk factors of Helicobacter pylori infection in Korea: Nationwide multicenter study over 13 years. BMC Gastroenterol. 2013 Jun 24;13:104. doi: 10.1186/1471230X-13-104.

27. Hu D, Shao J, Wang L, Zheng H, Xu Y, Song G, et al. Prevalence and risk factors of Helicobacter pylori infection in Chinese maritime workers. Ann Hum Biol. 2013Jun 25. [Epub ahead of print].

28. Nakajima S, Nishiyama Y, Yamaoka M, Yasuoka T, Cho E. Changes in the prevalence of Helicobacter pylori infection and gastrointestinal diseases in the past 17 years. J Gastroenterol Hepatol. 2010 May; 25Suppl 1:S99-S110. doi: 10.1111/j.1440-1746.2009.06214.x.

29. Shiota S, Mahachai V, Vilaichone RK, Ratanachu-ek T, Tshering L, Uchida T, et al. Seroprevalence of Helicobacter pylori infection and gastric mucosal atrophy in Bhutan, a country with a high prevalence of gastric cancer. J Med Microbiol. 2013Oct;62(Pt 10):1571-8. doi: 10.1099/ jmm.0.060905-0. Epub 2013 Jul 5.

30. Krashias G, Bashiardes S, Potamitou A, Potamitis GS, Christodoulou C. Prevalence of Helicobacter pylori cagA and vacA genes in Cypriot patients. J Infect Dev Ctries. 2013 Sep 16;7(9):642-50. doi: 10.3855/jidc.2923.

31. Bakri MM. Prevalence of Helicobacter pylori infection and the incidence of urea and clarithromycin resistance gene 23S rRNA genotypes status in Saudi Arabia. Saudi J Biol Sci. 2013 Jan;20(1):75-8. doi: 10.1016/j.sjbs.2012.10.006. Epub 2012 Nov 16.

32. Gonzalez-Carbajal M, Sevilla LF, Gra B. Alteraciones histológicas de la mucosa gástrica y prevalencia del Helicobacter pylori en pacientes dispépticos / Histologicalterations of the gastric mucous and prevalence of Helicobacter pylori in dyspeptic patients. Rev. Panam. Infectol. 2005;7(1):8-15.

33. Dooley CP, Cohen H, Fitzgibbons PL, Bauer M, Appleman MD, Perez-Perez GI, et al. Prevalence of Helicobacter pylori Infection and Histologic Gastritis in Asymptomatic Persons. N Engl J Med. 1989;321:1562-1566.

34. Mustapha SK, Bolori M, Ajayi N, Nggada H, Pindiga U, Gashau W, et al. Endoscopic findings and the frequency of Helicobacter pylori among dyspeptic patients in North Eastern Nigeria. Internet J Gastroenterology. 2007;6:1528-32.

35. Correa P. Human gastric carcinogenesis: A multistep and multifactorial process. First American Cancer Society award lecture on cancer epidemiology and prevention. Cancer Res. 1992;52:6735-40.

36. IARC Working Group on the Evaluation of Carcinogenic Risks to Humans. Schistosomes, liver flukes and Helicobacter pylori. In: World Health Organization. IARC Monographs on the Evaluation of Carcinogenic Risks to Humans. Lyon: 
World Health Organization; 1994. p. 177-220. (IARC Monographs on the Evaluation of Carcinogenic Risks to Humans; chapter 61).

37. Parsonnet J, Hansen S, Rodriguez L, Gelb AB, Warnke RA, Jellum E, et al. Helicobacter pylori infection and gastric lymphoma. N Engl J Med. 1994;330(18):1267.

38. Chang CS, Chen LT, Yang JC, Lin JT, Chang KC, Wang JT. Isolation of a Helicobacter pylori protein, FldA, associated with mucosa-associated lymphoid tissue lymphoma of the stomach. Gastroenterology. 1999;117(1):82.
39. Lin WC, Tsai HF, Kuo SH, Wu MS, Lin CW, Hsu PI, et al. Translocation of Helicobacter pylori CagA into Human B lymphocytes, the origin of mucosa-associated lymphoid tissue lymphoma. Cancer Res. 2010;70(14):5740.

40. Mysokerar VY, Chitralekha, Dandekar P, Prakash BS. Histopathological changes in acid peptic disease associated with Helicobacter pylori. Indian J Pathol Microbiol. 1992;23:548-56. 